\title{
A limited role for dual EGFR and ErbB2 inhibition in cholangiocarcinoma?
}

A preclinical study indicates that combinations of targeted therapies with erbB receptor tyrosine kinase inhibitors might have limited efficacy in patients with advanced cholangiocarcinoma. "Under particular conditions, dual targeting of EGFR and ErbB2 could be a potentially useful strategy for cholangiocarcinoma therapy, but such an approach is likely to be ineffective by itself against advanced cancer," says Alphonse Sirica, the study's corresponding author.

EGFR and/or ErbB2 are overexpressed in human and rodent cholangiocarcinoma cells. However, inhibitors of these tyrosine kinases, despite suppressing cholangiocarcinoma growth in cell culture and animal models, elicit a limited therapeutic response in patients with advanced disease.

The researchers investigated the effects of AG1517, an EGFR-specific tyrosine kinase inhibitor, and AG879, which targets
ErbB2, both singly and in combination, as well as those of lapatinib, a dual EGFR and ErbB2 inhibitor. "Simultaneous targeting of EGFR and ErbB2 signaling significantly suppresses the in vitro growth of ... cholangiocarcinoma cells," says Sirica. Combined treatment with AG1517 and AG879 was more effective than treatment with either agent alone, and lapatinib demonstrated even more potent effects than AG1517 plus AG879. In a rat model of cholangiocarcinoma, however, lapatinib did not inhibit tumor growth in animals with advanced disease, despite achieving a significant reduction in tumor growth in animals treated with this agent 2 days after intrahepatic tumor inoculation.

Shreeya Nanda

Original article Zhang, Z. et al. Preclinical assessment of simultaneous targeting of epidermal growth factor receptor (ErbB1) and ErbB2 as a strategy for cholangiocarcinoma therapy. Hepatology 52, 975-986 (2010) 